\title{
An Enhanced Approach on Projections on Climate and Land Analysis using ARIMA Modelling
}

\author{
Namratha $\mathrm{V}$ \\ Research Scholar, Civil Engineering Department, \\ UVCE, JB Campus, Bangalore University, \\ Bangalore, India.
}

\author{
Usha N Murthy \\ Professor and Head, Civil Engineering Department, \\ UVCE, JB Campus, Bangalore University, \\ Bangalore, India.
}

\begin{abstract}
Building up an association between provincial climatic fluctuation and precipitation conveyance changeability related with extraordinary occasions will empower us to increase an improved comprehension of the possible effects of environmental change on basic environmental changes -, for example, broadacre cultivating - by means of the precipitation conveyance system. Authentic concise information shows that ongoing atmosphere changeability shows more prominent disparity from the drawn out pattern, proposing that short-to-medium term atmosphere inconstancy can be examined utilizing the succinct qualities of the conveyance system.Hence, in the event that we are to comprehend the variety in precipitation conveyance and water accessibility related with environmental change and its expected effect on normal assets what's more, dependent human exercises -, for example, soil and horticulture - at that point we should consider the atmosphere uniqueness from long haul patterns, instead of the drawn out patterns themselves. This paper looks to exhibit that succinct characterization (in the meteorological setting) is a procedure that can be utilized to help us set up a association between atmosphere dissimilarity and deviations in precipitation examples, and along these lines can be adjusted as a powerful short-to medium term atmosphere expository and level representation.
\end{abstract}

Keywords - Meteorological Analysis, Air Depletion, air quality analysis, Metropolitan locations

\section{INTRODUCTION}

The effects of present and potential future environmental change present significant logical and cultural difficulties. Researchers have watched changes in temperature, ocean ice, and ocean level, also, credited those progressions to human action. It is a dire worldwide need to improve our comprehension of the atmosphere framework - a framework portrayed by complex wonders that are hard to watch and much more hard to mimic. Regardless of the expanding accessibility of computational assets, current diagnostic instruments have been outpaced by the ever-developing measures of watched atmosphere information from satellites, ecological sensors, and atmosphere model reenactments. Computational methodologies will in this manner be vital for these investigation challenges. The objective of the youngster research discipline, atmosphere informatics, is to move coordinated effort between atmosphere researchers what's more, information researchers (AI, insights, what's more, information mining scientists), and therefore connect the hole among information and comprehension. Exploration on atmosphere informatics will quicken revelation and answer squeezing inquiries in atmosphere science. AI is a functioning examination zone at the interface of software engineering and insights. The objective of AI research is to create calculations, robotized strategies, to identify designs in information. Such calculations are basic to a scope of advances including Web search, proposal frameworks, customized Internet publicizing, PC vision, and normal language preparing. AI additionally benefits the regular sciences, for example, science; the interdisciplinary bioinformatics field has encouraged numerous disclosures in genomics and proteomics. The effect of AI on atmosphere science has the possibility to be likewise significant. Here, we center explicitly around difficulties in atmosphere demonstrating; in any case, there are bunch coordinated efforts conceivable at the convergence of these two fields. Late work uncovers that joint efforts with atmosphere researchers additionally create intriguing new issues for AI.

Taking into account that electric foundation resources and systems are extensive and associated across parts what's more, require enormous open ventures, this investigation intends to give some refreshed data about the environmental change anticipated over Italy. The expansion of temperatures, the difference in precipitation designs, the expansion of dry spells in recurrence. Also, power just as the increase of other outrageous occasions have been examined so as to encourage the incorporated examination of future atmosphere impacts, vulnerabilities, adjustment, and alleviation and, accordingly, reinforce flexibility and versatile ability to atmosphere related perils for the Italian electric part.

The research is introduced as follows: informational indexes and systems received are introduced in Section II; conversation of the outcomes are attracted Section III; a few ends are given in Section IV

\section{LITERATURE REVIEW}

Worldwide Meteorological and climate Modelling design started in the 1970s. The models have gotten increasingly mind boggling as computational assets have developed. At present, around 25 research centers over the world bolster right around 50 atmosphere models, framing 
the premise of atmosphere projections (expectations) evaluated by the Intergovernmental Panel on Climate Change (IPCC), which was built up by the United Nations in 1988 and got the 2007 Nobel Peace Prize (imparted to previous VP Al Gore). Atmosphere researchers at first built up the Coupled Model Intercomparison Project variant 3 (CMIP3) document to bolster the IPCC Fourth Assessment Report.5 Scientists have utilized the chronicle in more than 500 distributions, and it's a rich wellspring of atmosphere reenactment yield. The CMIP5 venture proceeds with the custom of making worldwide atmosphere model forecasts simpler to utilize, and it will be very critical in future IPCC reports. The multi-model gathering (MME), the troupe of atmosphere models that advises the IPCC, has high difference over model forecasts, for an assortment of reasons. Various groups of researchers planned each model dependent on logical first standards, which prompted contrasts in scope, discretization suspicions, the included science, and coding mistakes.Despite the fact that the various models' forecasts differ There has been developing enthusiasm, in the atmosphere displaying network, in better approaches to join MME expectations, just as strategies to evaluate the "aptitude" of a solitary atmosphere model (measuring the model's precision over a predictable forecast). Analysts endeavoring to rank or weight models must show that the decisions are important for the particular setting. One methodology bolstered by the atmosphere science network is the ideal model supposition. In this structure, analysts accept one model to be "reality," at that point, over an adjustment stretch, they assess expectation strategies prepared on mimic perceptions created by the "valid" model. Researchers talked about these issues at an IPCC Expert Meeting on Assessing and Combining Multi-Model Climate Projections.

\section{RESEARCH METHODOLOGY}

The fundamental manner by which inadequate observational information is utilized to develop total fields is through information digestion. This field of exploration concerns how to refresh material science based models with watched information, and incorporates such innovation as the troupe Kalman filter.

Information aggregation in a staple of climate conjectures, and the different re-investigations in the air and sea. From multiple points of view, this is the most refined utilization of the mix of models and perceptions, however its utilization in improving atmosphere expectations is still in its early stages. For climate time scales, this works well. For longer-term estimates (seasons to decades) the key factors are in the sea, not the environment; atmosphere researchers still can't seem to completely build up an atmosphere model initalization in which the development of sea fluctuation models this present reality in helpful ways. Climate researchers locate the early outcomes fascinating, if not persuading, and a lot more models are scheduled to come online in the new CMIP5 archive. 20

Advances in information osmosis could likewise profit different zones of software engineering. In mechanical technology, for instance, when a robot utilizes a material science based model for the elements administering its development, frequently it should likewise fuse data gathered from the locally available sensors, and, in a few cases, extra continuous directions from a human controller.

Creating and Understanding Perturbed Particle Ensembles (PPE) The spread among various model forecasts from various displaying bunches is one approach to measure the models' "basic vulnerability". It may, we can't consider these models a controlled arbitrary example from the space of every single conceivable model. A methodology that prompts an all the more precisely portrayed outfit is to take a solitary model, and fluctuate numerous (dubious) boundaries inside the code, creating a group of comparative models that in any case test a decent arrangement of the inherent vulnerability that emerges when we pick a particular arrangement of boundary esteems. Scientists have effectively utilized these Perturbed Particle Ensembles (PPEs) in the Climateprediction.net and Quantifying Vulnerability in Model Predictions (QUMP) tasks to produce controlled model groups that they can deliberately contrast with watched information, and afterward make inferences.

Be that as it may, planning such investigations and effectively dissecting in some cases a great many recreations is a test, yet one which is progressively going to be endeavored. We trust that this article empowers future work, not just on a portion of the test issues proposed here, yet additionally on new issues in atmosphere informatics. An enormous and fluctuated measure of atmosphere information is accessible, giving a rich and ripe play area for future AI and information mining research. Indeed, even exploratory information examination could demonstrate helpful for quickening disclosure. There are innumerable joint efforts conceivable at this crossing point of atmosphere science and AI, information mining, and measurements. We firmly support future advancement on a scope of rising issues in atmosphere informatics

\section{RESULTS AND DISCUSSION}

The consequences of the best model for the whole perception arrangement. Precipitation changes are significantly more spatially heterogeneous contrasted and temperature change. In winter the all out precipitations are anticipated to increment over North Italy from 5\% to $30 \%$ throughout the century, while during summer the precipitations are probably going to diminish of about $5 \%$, $10 \%$ till half separately in FUT1, FUT2 and FUT3 periods. Some coast regions will be influenced by an expansion of precipitations with values relying upon locale and season, specifically during winter and harvest time. Fig. 4 As for Fig. 3 however for absolute precipitation oddities expessed in $\%$ values regard to add up to precipitation esteems in the reference time frame 


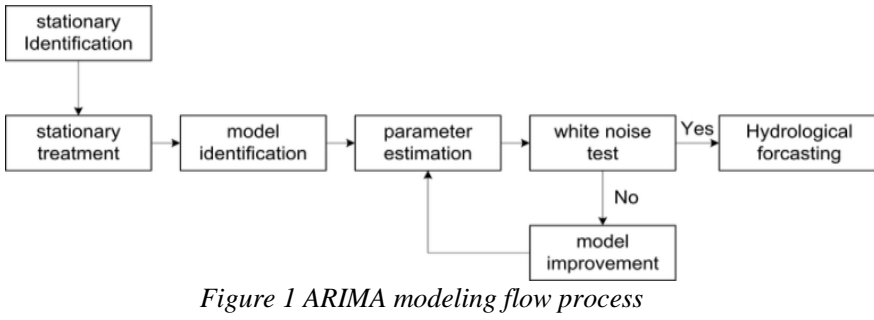

This is huge, in light of the fact that just looking back can one decide the best model for the entire perception succession. We utilized web based learning calculations with the objective of making both continuous what's more, future expectations. Besides, our examination shows that the gullible "group" approach has weaknesses due to the non-stationary idea of the perceptions and the generally short history of model expectation information. Atmosphere researchers use temperature abnormalities to communicate both the atmosphere model forecasts and the genuine perceptions. A temperature inconsistency is the distinction between the watched temperature and the temperature at the equivalent area at a fixed, benchmark time. For instance, in a specific month it may be $80^{\circ} \mathrm{F}$ in Delhi, and $70^{\circ} \mathrm{F}$ in Bangalore, however the inconsistency from the benchmark time may be $1^{\circ} \mathrm{F}$ in the two spots. Hence, fluctuation is lower at the point when specialists normal temperature peculiarities over numerous geographic areas, than when they utilize total temperatures. Figure 2 shows atmosphere model recreation runs, and perception information, arrived at the midpoint of over numerous geological areas, and ordinarily in a year, yielding one esteem for a worldwide mean temperature peculiarity every year.

The outcomes acquired are on ARIMA Modelling algorithm,[10] which tracks a moving arrangement of temperature esteems concerning "master" expectations, which we used to speak to the atmosphere models. This presents a calculation that learns the exchanging rate between best specialists, while at the same time performing the first expectation task.Also there's no perception information later on with which we could assess the AI calculations. To uncover the qualities of CPP affected by the atmosphere factors at a sub-territorial scale, the examination zone is partitioned into 8 sub-districts dependent on SPEI12 utilizing the PCA what's more, the Variable max pivot techniques.

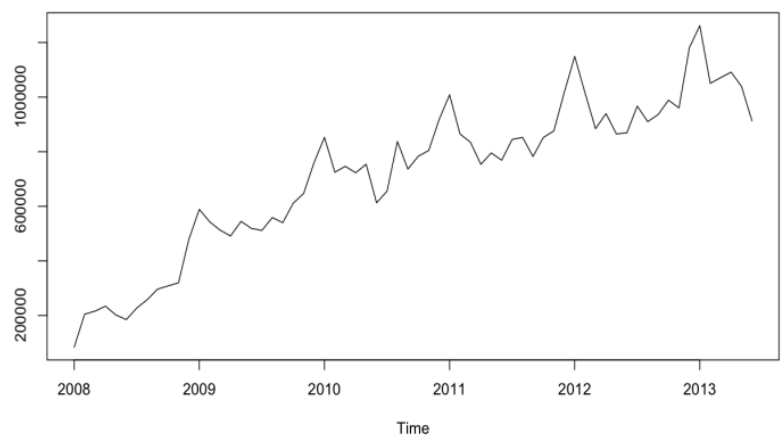

Figure 2 ARIMA modeling based Regional plot Analysis
The primary goals of my research are twofold: right off the bat, to build up an association between atmosphere disparity and precipitation conveyance fluctuation utilizing brief characterization systems; and also, to build up a successful and transportable diagnostic and hazard appraisal/determining instrument that will be an indispensable piece of a choice help toolbox to advise venture, adjustment, asset condition appraisal, also, land and water the board.

The Research is self-centered around two broad acre cultivating zones as contextual investigations; the dry land and inundated horticultural sub-areas of southwestern India (SWI) and a practically identical area in North America. The SWA contextual investigation is being utilized to build up the association between atmosphere disparity and precipitation inconstancy, and afterward to build up the atmosphere logical and determining instrument.

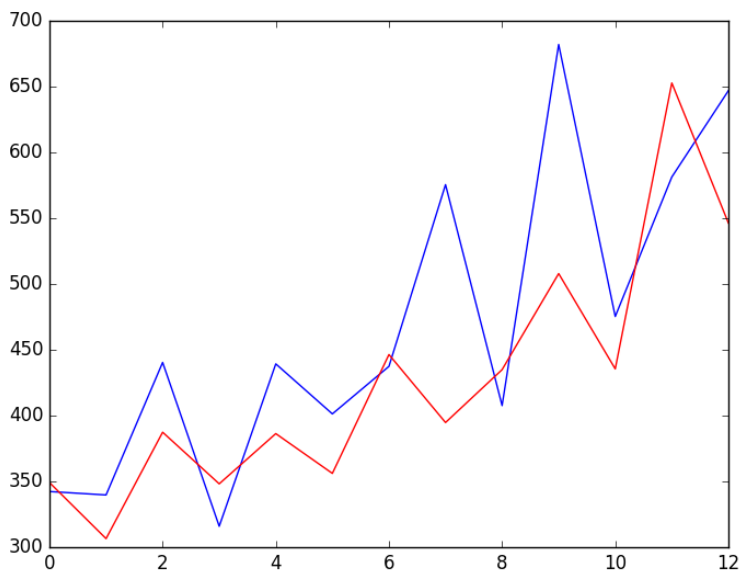

Figure 3. Variation Plot Analysis(Month wise Analysis on Land depletion based approach)

The North Indian contextual analysis will at that point be used to show that concise characterization can be applied to comprehend and survey the dangers related with the expected effects of short-to-medium term precipitation fluctuation at a given area where sufficient succinct information is accessible. These contextual investigations will likewise give the premise to device approval as chosen agent territories in danger of common and outrageous reactions to environmental change.

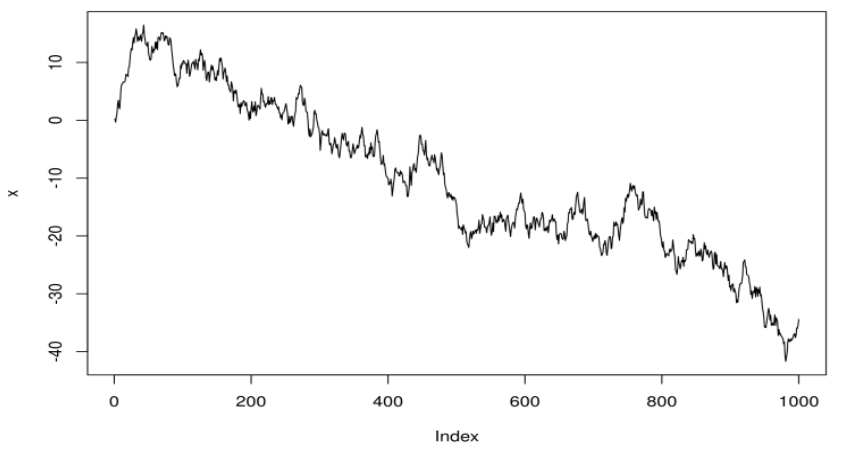

Figure 4. Index vs dB based Analysis 
Precipitation is the overwhelming component controlling the spatial dissemination of CPP, particularly in the semibone-dry locales in North India, which is in acceptable understanding with others' examinations dependent on the field perceptions [32].

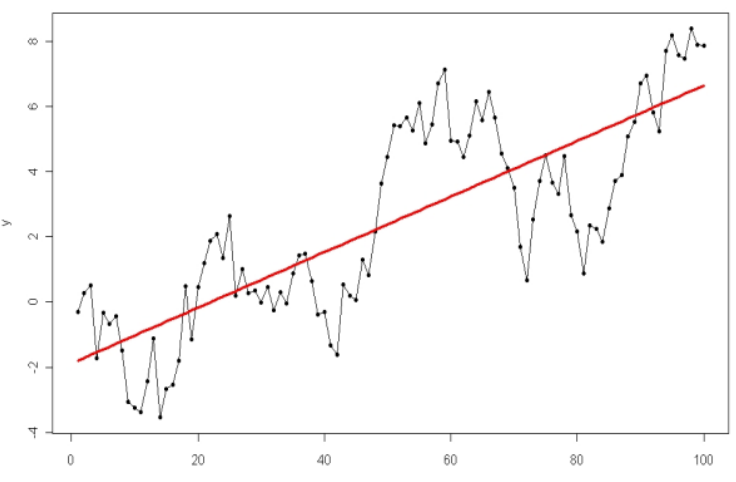

Figure 5 Region of Convergence Plot vs Linearity Analysis

Moreover, sensor innovations are being created to praise the atmosphere scientific and determining device, which will give contribution to the dynamic model to assess the possible outcomes and redistributions of supplements, and in this manner the flexibility of the framework because of progress. This represents an elective perspective on strength, that is: "a framework's capacity to ingest unsettling influences and redesign itself into a superior arrangement, while holding its essential qualities" [24].

By embracing this view we begin to see the utilization of dynamic demonstrating in a catchment, biological system and worldwide setting, for example, I proposed prior and as showed in the CSIRO model above [25],[26].

\section{CONCLUSION}

Applying ARIMA modelling to issues encompassing water, vitality, and food security will at last energize the advancement of improved land and water quality administration plans and little and enormous scope choice emotionally supportive networks. Together, these plans and frameworks will give increased levels of trust in foreseeing water quality under changing atmosphere situations. Notwithstanding, this requires the improvement of new apparatuses and innovations to permit us to make the important water and contaminant motion estimations more delegate and practical than has so far been the situation. Horrible climatic conditions and the debasement of normal assets are the primary requirements influencing farming profitability [27].

In this Approach, an improved comprehension of precipitation inputs and the cooperation among surface, and subsurface spillover and the re-dissemination of supplement and contaminants will be a noteworthy advance forward. By evaluating conveyance and transport components more noteworthy comprehension of the elements of catchment frameworks can be picked up, and in this way of the environment merchandise and enterprises gave. On the off chance that scene reaction times and motion changes in transportation are caught by new and inventive innovations, at that point they can be connected with concise characterization models to give a viable 2015 second International Conference on Signal Processing and Integrated Networks (SPIN) atmosphere investigation and anticipating device that land and water directors can use for recognizing and examining the hazard and estimating the expected effect of atmosphere difference and short-tomedium term precipitation fluidly [28].

Thus, land without depletion, water the executives results can be accomplished - these results are the essential drivers for my examination. An underlying audit of characterization approach applied in the meteorological setting shows that succinct characterization - as talked about and characterized in this - can be used to help us set up an association between atmosphere uniqueness and precipitation conveyance inconstancy, and along these lines be adjusted as a powerful short-to-medium term atmosphere systematic and estimating instrument.

The improvement of such an instrument, along with better observing advances and information assortment choices, will give a system to better dynamic and hazard the executives. This is at the core of the nexus way to deal with dealing with the dynamic idea of between related objectives and assets, the fundamental understanding that choices in a single segment will influence another. Without this kind of all encompassing methodology, giving secure practical access to water and food, while tending to our vitality needs, This Approach would be ideally significant to save the nature in a better way.

\section{REFERENCES}

[1] Y. Zhang, C. Zhang, Z. Wang, Y. Chen, C. Gang, R. An, and J. Li, "Vegetation dynamics and its driving forces from climate change and human activities in the three-river source region, China from 1982 to 2012,', Sci. Total Environ., vols. 563-564, pp. 210-220, Sep. 2016.

[2] Y. Gao, X. Zhou, Q. Wang, C. Wang, Z. Zhan, L. Chen, J. Yan, and R. Qu, "Vegetation net primary productivity and its response to climate change during 2001-2008 in the Tibetan Plateau," Sci. Total Environ., vol. 444, pp. 356-362, Feb. 2013.

[3] F. Chen, A. Lin, H. Zhu, and J. Niu, "Quantifying climate change and ecological responses within the yangtze river basin, China,' Sustainability, vol. 10, no. 9, p. 3026, Aug. 2018.

[4] D. Wu, X. Zhao, S. Liang, T. Zhou, K. Huang, B. Tang, and W. Zhao, "Time-lag effects of global vegetation responses to climate change," Glob Change Biol., vol. 21, no. 9, pp. 3520-3531, Sep. 2015.

[5] C. Monteleoni et al., "Tracking Climate Models," Statistical Analysis and Data Mining, vol. 4, no. 4, 2011, pp. 372-392.

[6] C. Monteleoni et al., "Climate Informatics," Computational Intelligent Data Analysis for Sustainable Development; Data Mining and Knowledge Discovery Series, CRC Press, 2013, pp. 81-126.

[7] S. McQuade and C. Monteleoni, "Global Climate Model Tracking Using Geospatial Neighborhoods," Proc. 26th AAAI Conf. Artificial Intelligence, AAAI, 2012, pp. 335-341.

[8] G.A. Schmidt et al., "Present Day Atmospheric Simulations Using GISS ModelE: Comparison to InSitu, Satellite and Reanalysis Data,' J. Climate, 2006, vol. 19, no. 2, pp. 153-192. CISE-15-5Monteleoni.indd 39 23/10/13 3:52 PM 40 Computing in Science \& Engineering 
[9] S. Solomon et al., Climate Change 2007: The Physical Science Basis. Contribution of Working Group I to the Fourth Assessment Report of the Intergovernmental Panel on Climate Change, Cambridge Univ. Press, 2007.

[10] T. Reichler and J. Kim, "How Well Do Coupled Models Simulate Today's Climate?," Bull. Am. Meteorological Soc., vol. 89, no. 3, 2008, pp. 303-311; http://dx.doi.org/10.1175/BAMS-89-3-303.

[11] C. Reifen and R. Toumi. "Climate Projections: Past Performance No Guarantee of Future Skill?” Geophysical Research Letters, vol. 36, no. 13, 2009; doi:10.1029/2009GL038082.

[12] M. Brunetti , M.Maugeri, F. Monti, T. Nanni (2004) "Changes in daily precipitation frequency and distribution in Italy over the last 120 years, J. Geophys. Res., 109, doi:10.1029/2003JD004296.

[13] P. Faggian, "Climate Change projections for Mediterranean Region with focus over Alpine". Journal of Environmental Science and Engineering vol. B 4, n. doi:10.17265/2162-5263/2015.09.004, pp. 482-500, 2015.

[14] R. Bonanno, P. Faggian, "Changes in Precipitation Regime over Italian Peninsula and their possible impacts on the Electric System", Tethys. 2018, 15, 18-30 www.tethys.cat ISSN-1697-1523 eISSN1139-3394 DOI:10.3369/tethys.2018.15.

[15] M. Brunetti , M.Maugeri, F. Monti, T. Nanni (2006) “Temperature and precipitation variability in Italy in the last two centuries from homogenised instrumental time series, Int. J.Climatol., 26, 345-381.

[16] G. Forzieri, L. Feyen, S. Russo, M. Vousdoukas, L. Alfieri, S. Outten, M. Migliavacca, A. Bianchi, R. Rojas, A. Cid, "Multi-hazard assessment in Europe under climate change". Clim Change 2016, vol 137, pp105-119. https ://doi.org/10.1007/s1058 4-016-1661-x.

[17] DOE, "Energy Sector Vulnerabilities to climate Change and Extreme Weather “ DOE/PI-0013, 2013

[18] P.Faggian, R. Bonanno (2018) "Raising awareness on climatechange related hazards that might impact electric infrastructures" 978-8- 8872-3740-5@2018 AEIT

[19] P.Faggian, R. Bonanno, M. Lacavalla, P. Marcacci, G. Pirovano (2018) "Research and operational activities to cope with wet snow impacts on overhead power lines in current and future climate over Italy" 2nd South East European Regional CIGRE Conference, Kyiv 2018

[20] Jacob et al. "EURO-CORDEX: new high-resolution climate change projections for European impact research" Regional Environmental Change,, vol. 14, n. I2, pp. 563-578, 2014

[21] Detlef P et al (2011) The representative concentration pathways: an overview. Clim Change 109:5-31. https//doi.org/10.1007/s10584011-0148-z

[22] M.R. Haylock et al.“A European Daily High-Resolution Gridded Dataset of Surface Temperature and Precipitation." J. Geophys. Res (Atmospheres) 113: D20119. doi:10.1029/2008JD10201

[23] T. Landelius, P. Dahlgren, S. Gollvik, A. Jansson, E. Olsson, “A high-resolution regional reanalysis for Europe. Part 2: 2D analysis of surface temperature, precipitation and wind,» Q. J. R. Meteorol. Soc., vol. 142, p. 2132-2142, 2016.

[24] Meehl, G. A. 2005. "Overview of the Coupled Model ntercomparison Project.” Bull. Am. Meteorol. Soc. 86:89-93.

[25] WMO, "Guidelines on Analysis of extremes in a changing climate in support of informed decisions for adaptation. Technical Report" WCDMP No. 72, WMO/TD-No. 1500 , Geneva, Switzerland., 2009.

[26] M.A. Hernández-Ceballos, H. Garcia-Mozo, J.A. Adame, E. Dominquez-Vilches, B.A. De la Morena, J.P. Bolivar and C. Galán, "Synoptic and meteorological characterisation of olive pollen transport in Córdoba province (south-western Spain)", International Journal of Biometeorology, 2011; vol. 55, iss. 1, pp 17-34. [online] URL: http://link.springer.com/article/10.1007\%2Fs00484-010-0306-

[27] M.F. McCabe, H. Gao and E.F. Wood (2004) "An Evaluation of Soil Moisture Predictions Derived from AMSR-E using Ground Based, Airborne and Ancillary Data During SMEX 02", Department of Civil and Environmental Engineering, Princeton University, Princeton, NJ 08544, USA, April 2004. [online] URL: http://hydrology.princeton.edu/ mmccabe/Eric/McCabe-SMEX02V2.doc

[28] M.V.K. Sivakumar and R.P. Motha, Managing Weather and Climate Risks in Agriculture, Springer Science \& Business Media, 2008, p.451.

[29] V. Mircov, C. Moisa, L. Nita and A. Cozma A. "Climatological and Synoptic Characterisation during winter 2009-2010." Research
Journal of Agriculture Science, vol. 43, no. 3, pp 437-442, Faculty of Agriculture, Timisoara, 2011. [online]

[30] Z. Bielec, "Przebieg dobowy i charakterystyka synoptyczna burz w Krakowie w latach 1896-1995 [Daily course and synoptic characterisation of thunderstorms in Cracow in the period 18961995]." Zeszyty Naukowe Uniwersytetu JagielloĚskiego, Prace Geograficzne, no. 105, pp. 73-83, Kraków 2000. [online] URL http://www.pg.geo.uj.edu.pl/documents/3189230/4676055/2000_10 5_73-84.pdf

[31] United States Geological Survey (USGS), "Climatic Interpretations of Terrestrial Paleoecology, Study Plan Number R 3 1.5.13A, Rev 0", January 13 1992, p4-1, Prepared by USGS for the U.S Department of Energy, Office of Civilian Radioactive Waste Management, Washington, DC 2O85.[online]URL:http://pbadupws.nrc.gov/docs/ML0318/ML0318 40511.pdf 